\title{
Koinzidenz oder Kausalität?
}

Fragestellung: Stellt das Auftreten einer Depression in bestimmten Lebensphasen ein Risiko für eine Alzheimer-Demenz (AD) und/oder vaskuläre Demenz (VaD) dar?

Hintergrund: Depression ist eine häufige Komorbidität beider Demenzformen. Bisher ist unklar, ob dies Ausdruck einer engen pathophysiologischen Verflechtung neurobiologischer Mechanismen ist, ob eine Depression als Prodromalzustand einer AD oder VaD auftritt oder eine ätiologische Bedeutung für die Demenzentstehung hat. Das Auftreten depressiver Syndrome könnte zudem bei beiden Demenzformen eine unterschiedliche Wertigkeit haben. Insbesondere für vaskuläre Erkrankungen und Depression wird eine gegenseitige Verstärkung angenommen.

Patienten und Methodik: In einer retrospektiven Kohortenstudie wurden die Daten von Teilnehmern eines privaten Gesundheitsfürsorgeprogramms während der Jahre 1964 bis 1973 (damals 40 bis 55 Jahre alt) analysiert. Ermittelt wurde unter anderem, ob stationäre Behandlun-

Barnes DE, Yaffe K, Byers AL et al. Midlife vs late-life depressive symptoms and risk of dementia: differential effects for Alzheimer disease and vascular dementia. Arch Gen Psychiatry 2012; 69: $493-8$ gen aufgrund einer MajorDepression oder Dysthymie nach ICD-9 in den Jahren 1964 bis 1973 (Depression in mittlerer Lebensphase) und/oder in den Jahren 1994 bis 2000 (Depression in später Lebensphase) durchgeführt und diese mit dem Auftreten einer Demenz beziehungsweise differenziell einer $\mathrm{AD}$ oder VaD in 2003 bis 2009 in Beziehung gesetzt wurden.

Ergebnisse: Ausgewertet wurden Daten von 13.535 Personen im Alter von $81 \pm 4,5$ Jahren im Jahr 2009. Depressiv nur im mittleren Lebensabschnitt waren hiervon $14,1 \%$, nur im späten Lebensabschnitt 9,2\% und in beiden Lebensphasen 4,2\%. Während des sechsjährigen Follow-up erhielten 22,5\% die Diagnose einer Demenz (AD: 5,5\%, VaD: 2,3\%). Das adjustierte Demenzrisiko bei Vorliegen depressiver Symptome nur im mittleren Lebensabschnitt lag bei $20 \%$ (Hazard Ratio [HR] = 1,19; $95 \%$-Konfidenzintervall [KI] 1,07-1,32), nur im späten Lebensabschnitt bei $70 \%$ $(\mathrm{HR}=1,72,95 \%$-KI 1,54-1,92) und für beide Lebensphasen bei $80 \%(\mathrm{HR}=1,77,95 \%-\mathrm{KI}: 1,52-2,06)$. Es zeigte sich eine Verdopplung des Risikos für eine AD bei Personen mit depressiven Symptomen im späten Lebensabschnitt $(\mathrm{HR}=2,06,95 \%-\mathrm{KI}$ $1,67-2,55)$. Dagegen wurde für die VaD eine Verdreifachung des Risikos bei Personen mit Depressivität im mittleren und späten Lebensalter nachgewiesen ( $\mathrm{HR}=3,51,95 \%$-KI 2,44-5,05).

Schlussfolgerungen: Depressivität in der mittleren und späten Lebensphase sind mit einem erhöhten Demenzrisiko vergesellschaftet. Bei Patienten mit Depression im höheren Lebensalter könnte es sich eher um ein Prodrom einer AD handeln, wenn eine Erstmanifestation vorliegt, während eine wiederholte Manifestation zu einer VaD prädisponiert und Ausdruck eines ätiologischen Zusammenhangs sein könnte.

\section{-Kommentar von PD Dr. Christian Lange-Asschenfeldt}

\section{Depressionen erhöhen auch das Risiko einer Alzheimer-Demenz}

Die Autoren heben sehr auf ihren Befund ab, dass rezidivierende Depressionen das Risiko für eine $\mathrm{VaD}$ 3,5-fach erhöhen und sehen dies als Beleg für einen ätiopathogenetischen Zusammenhang. Die Studie zeigt aber zusätzlich, dass rezidivierende Depressionen auch das AD-Risiko erhöhen, was in dem Paper erstaunlicherweise unkommentiert bleibt. Dies aber zeigt, dass auch zwischen rezidivierender Depression und AD ein ätiologischer Zusammenhang bestehen könnte. Vermutet wird seit langem eine Schädigung hippokampaler Neurone durch Hyperkortisolämie infolge depressionsbedingter chronischer Dysregulation der Hypothalamus-Hypophysen-Nebennierenrinden-Achse. Dass depressive Patienten allgemein ein erhöhtes Risiko haben, eine Demenz zu entwickeln, ist ein gut gesicherter Befund. Bemerkenswert ist, dass in den größten Kohortenstudien die Zahlen hierzu nahezu identisch sind (aktuelle Studie: $\mathrm{HR}=1,77$, Beobachtungszeitraum > 40 Jahre; Framingham-Studie: $\mathrm{HR}=1,72$, Beobachtungszeitraum 17 Jahre; Li et al.: $\mathrm{HR}=1,71$ Beobachtungszeitraum 15 Jahre). Befunde dieser Art haben eine wichtige prognostische und sozialmedizinische Implikation im (noch nicht evidenzbasierten, aber intuitiv naheliegenden) Umkehrschluss: Eine konsequente antidepres- sive Behandlung von rezidivierenden depressiven Syndromen bereits in der mittleren Lebensphase könnte das Risiko einer späteren Demenzentwicklung senken, und zwar nach den Daten der vorliegenden Studie vor allem der VaD, aber eben auch der AD. Schließlich ist mit den Autoren zu folgern, dass hinter depressiven Erstmanifestationen im Alter immer auch eine beginnende Demenz vermutet und abgeklärt werden sollte.

\section{Literatur beim Verfasser}

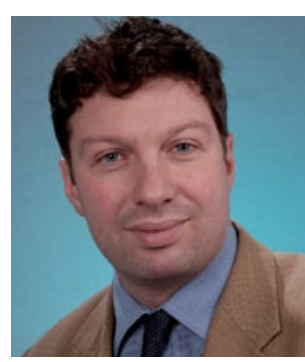

PD Dr. med. Christian LangeAsschenfeldt, Düsseldorf

Klinik und Poliklinik für Psychiatrie und Psychotherapie der Heinrich-HeineUniversität, LVR-Klinikum Düsseldorf E-Mail:kn50050@lvr.de 